\title{
Identification of key genes associated with bladder cancer using gene expression profiles
}

\author{
YUPING HAN $^{1}$, XUEFEI JIN ${ }^{1}$, HUI ZHOU ${ }^{2}$ and BIN LIU ${ }^{1}$ \\ Departments of ${ }^{1}$ Urology and ${ }^{2}$ Gastrointestinal Colorectal and Anal Surgery, \\ China-Japan Union Hospital of Jilin University, Changchun, Jilin 130033, P.R. China
}

Received August 12, 2016; Accepted August 23, 2017

DOI: $10.3892 / \mathrm{ol} .2017 .7310$

\begin{abstract}
The aim of the present study was to further investigate the molecular mechanisms of bladder cancer. The microarray data GSE52519 were downloaded from Gene Expression Omnibus, comprising 9 bladder cancer and 3 normal bladder tissue samples. Differentially expressed genes (DEGs) were identified using Limma package analysis. Subsequently, Gene Ontology, Kyoto Encyclopedia of Genes and Genomes and Reactome pathway enrichment analyses were performed for down- and upregulated DEGs. Transcription factors and genes associated with cancer from DEGs were identified. Protein-protein interaction (PPI) networks were constructed using STRING, and pathway enrichment analysis was also conducted for genes in the core sub-network that was identified using BioNet. In total, 420 downregulated and 335 upregulated DEGs were identified. Functional and pathway enrichment analyses identified that a number of DEGs, including $A U R K A$, $C C N A 2, C C N E 1, C D C 20$ and $C C N B 2$, were enriched in the cell cycle. Furthermore, a total of 12 upregulated proto-oncogenes were identified, including AURKA and CCNA2. In the PPI sub-network, a number of DEGs (e.g., $C C N B 2, C D C 20$, $C C N A 2$ and $M C M 6)$ with higher degrees were enriched in the KEGG pathway of the cell cycle. In conclusion, the DEGs associated with the cell cycle (e.g., CDC20, CCNA2, CCNB2 and $A U R K A$ ) may serve pivotal roles in the pathogenesis of bladder cancer.
\end{abstract}

Correspondence to: Dr Xuefei Jin, Department of Urology, China-Japan Union Hospital of Jilin University, 126 Xiantai Street, Changchun, Jilin 130033, P.R. China

E-mail: xuefeijinxfj@sina.com

Abbreviations: CIN, chromosomal instability; GO-BP, Gene Ontology-Biological Process; KEGG, Kyoto Encyclopedia of Genes and Genomes; DEGs, differentially expressed genes; DAVID, Database for Annotation, Visualization and Integrated Discovery

Key words: bladder cancer, differentially expressed genes, pathway, network

\section{Introduction}

Bladder cancer is one of the most common types of cancer worldwide and was the most common urological tumor in China in 2012 (1). In 2012, of the individuals with bladder cancer, $90 \%$ were diagnosed at $>65$ years-of-age worldwide (2). This disease is a common malignancy characterized by a poor clinical outcome (3); therefore, investigations into the underlying molecular mechanisms are urgently required in order to facilitate improvements in early diagnosis and treatments.

Bladder cancer is considered a genetic disease and is driven by the multistep accumulation of genetic and epigenetic factors that usually result in uncontrolled cellular proliferation, cell cycle deregulation or a decrease in cell death (3). The two different types of genetic alterations that are observed in bladder cancer are tumor protein p53 mutations and a number of single-nucleotide and structural variants, as well as chromosome shattering (4). Di Pierro et al (1) revealed that mutations in FGFR3 and TP53 are usually predictive of bladder malignancy. Overexpression of PIN2/TRF1-interacting telomerase inhibitor 1 in urothelial carcinoma of the bladder inhibited cell proliferation by inhibiting telomerase activity and the p16/cyclin D1 signaling pathway (5). Furthermore, chromosomal instability (CIN) characterized by loss or gain of chromosomal fragments or entire chromosomes is most prevalent in invasive urothelial cancer, compared with other less malignant papillary subtypes (6). Checkpoint dysfunction serves an important role in the development of CIN and is caused by defects in cell cycle regulation, p53 function and checkpoint signaling (7). Although certain studies have reported that gene mutations, telomerase activity and chromosomal instability are connected with bladder cancer (4-7), the exact molecular mechanism of bladder cancer remains unclear. A profound understanding of the molecular mechanism of action may be useful to provide an improved, more efficient handling of bladder cancer.

In the present study, the raw microarray data GSE52519 were downloaded to investigate the underlying molecular mechanisms of bladder cancer. Gene Ontology-Biological Process (GO-BP) functional analysis, Kyoto Encyclopedia of Genes and Genomes (KEGG) pathway analysis and Reactome pathway enrichment analysis were performed for down- and upregulated differentially expressed genes (DEGs). Subsequently, transcription factors and genes associated with cancer for DEGs were identified. In addition, a protein-protein interaction (PPI) 
network and its core sub-network were constructed, and KEGG pathway enrichment analysis of the genes in the identified core PPI sub-network was also performed.

\section{Materials and methods}

Microarray data and data preprocessing. The raw microarray data GSE52519 were downloaded from Gene Expression Omnibus (www.ncbi.nlm.nih.gov/geo/query/acc. cgi?acc $=$ GSE52519). GSE52519 comprises 9 bladder cancer tissue samples obtained during cystectomy and 3 normal tissue samples derived from post mortem donors without bladder cancer. The microarray platform of GSE52519 was GPL13497 Illumina HumanWG-6 v3.0 Expression BeadChip (Illumina, Inc., San Diego, CA, USA).

The gene expression profile was preprocessed using Limma (version 3.83; linear models for microarray data; www.bioconductor.org/packages/2.8/bioc/html/limma.html) package in Bioconductor (8) and Affymetrix annotation files from Brain Array Lab (version 20; http://brainarray.mbni. med.umich.edu/Brainarray/Database/CustomCDF/CDF_ download.asp) (9). The background correction, quantile normalization and probe summarization of the microarray data were performed using the Robust Multi-Array Average algorithm (10) to obtain the gene expression matrix.

Identification of DEGs. The normalized data were calculated with the Limma package (8), and genes with $\mathrm{P}<0.01$ and $\mid \log _{2}$ fold changel $\geq 2$ were considered to indicate a statistically significant difference between the bladder cancer group and the normal group.

Enrichment analysis of DEGs. The Database for Annotation, Visualization and Integrated Discovery (DAVID; david. abcc.ncifcrf.gov) is a comprehensive functional annotation tool $(10,11)$. Based on DAVID online analysis, GO-BP functional enrichment analysis was performed for DEGs (12); $\mathrm{P}<0.01$ was selected as the threshold criterion.

KEGG is a knowledge base for systematic analysis of gene functions, and the PATHWAR database (www.kegg. $\mathrm{jp} / \mathrm{kegg} /$ pathway.html) is supplemented by the information of conserved sub-pathways (13). The Reactome database (www. reactome.org) is an open-source open-data resource of human pathways and reactions (14). Pathway enrichment analysis was performed for the DEGs using these two databases, with the threshold of $\mathrm{P}<0.01$.

Identification of transcription factors and genes associated with cancer from DEGs. Transcription factor analysis using the TRANSFAC database (www.biobase-international. com/product/transcription-factor-binding-sites\#resources) (15) was performed on DEGs to determine whether the genes were transcription factors. The DEGs were also submitted to the Tumor Suppressor Gene database (bioinfo.mc.vanderbilt. edu/TSGene) (16) and the Tumor-Associated Gene database (blog.synopse.info/tag/Database) (17) to obtain all known proto-oncogenes and tumor suppressor genes.

Construction of PPI network and PPI sub-network analysis. DEGs were submitted to STRING version 9.1 (Search Tool for the Retrieval of Interacting Genes; string.embl.de) (18) to search interaction associations of the proteins; the confidence score $>0.9$ was used as the threshold criterion. Then, visualization of the PPI network was performed using Cytoscape software (cytoscape.org) (19). The HUB nodes with the top 5 degrees in the PPI network were also obtained.

The PPI sub-network of DEGs was identified by BioNet (20), and a false discovery rate of $<0.01$ was selected as the threshold criterion. The pathway enrichment analysis of genes in the core PPI sub-network was performed using the KEGG database, and $\mathrm{P}<0.01$ was selected as the threshold criterion.

\section{Results}

DEG analysis. A total of 779 transcripts and 755 DEGs were identified in the bladder cancer and the normal group combined, including 431 downregulated transcripts that corresponded to 420 downregulated DEGs, and 348 upregulated transcripts that corresponded to 335 upregulated DEGs.

Enrichment analysis of DEGs. The top three enriched GO terms in the BP category of downregulated DEGs included muscle contraction $\left(\mathrm{P}=3.60 \times 10^{-5}\right)$, involving DEGs such as $C R Y A B, T A C R 2$ and $M Y H 3$, muscle system process $\left(\mathrm{P}=9.48 \times 10^{-5}\right)$ associated with DEGs such as $C R Y A B, T A C R 2$ and $M Y H 3$, and actin filament-based process $\left(\mathrm{P}=3.19 \times 10^{-4}\right)$ involving DEGs such as DLCl, MYH3 and CALDI (Table I). The only two enriched KEGG pathways of downregulated DEGs included focal adhesion $\left(\mathrm{P}=3.86 \times 10^{-3}\right.$; e.g., $P R K C A$, LAMA3 and ITGA8) and tight junction $\left(\mathrm{P}=8.10 \times 10^{-3}\right.$; e.g., PRKCA, GNAII and MYH3) (Table II). Additionally, five downregulated DEGs (TNNT3, DES, MYH3,DMD and TPM2) were significantly enriched in the Reactome pathway of muscle contraction $\left(\mathrm{P}=2.46 \times 10^{-3}\right.$; Table III).

The top three enriched GO terms in the BP category of upregulated genes included cell cycle $\left(\mathrm{P}=1.88 \times 10^{-29}\right)$, involving DEGs such as AURKA, CCNA2, CCNE1, CDC20 and $C C N B 2, \mathrm{M}$ phase $\left(\mathrm{P}=1.88 \times 10^{-29}\right)$ associated with a number of DEGs, including AURKA, CCNA2, CDC20 and CCNB2, and cell cycle phase $\left(\mathrm{P}=2.89 \times 10^{-28}\right)$ associated with $D E G$ such as AURKA, CCNA2, CCNE1, CDC20 and CCNB2 (Table IV). A total of six pathways were significantly enriched for upregulated genes, including cell cycle $\left(\mathrm{P}=6.68 \times 10^{-11}\right.$; e.g., $C C N A 2$, $C C N E 1, C D C 20$ and $C C N B 2)$, oocyte meiosis $\left(\mathrm{P}=1.76 \times 10^{-7}\right.$; e.g., AURKA, CCNE1, $C D C 20$ and $C C N B 2$ ), DNA replication $\left(\mathrm{P}=3.43 \times 10^{-4}\right.$; e.g., RFC4 and POLE2) and p53 signaling pathway $\left(\mathrm{P}=4.28 \times 10^{-4}\right.$; e.g., $C C N E 1$ and $\left.C C N B 2\right)$ (Table V). The five enriched Reactome pathways of upregulated genes included cell cycle, mitotic $\left(\mathrm{P}=3.93 \times 10^{-22}\right.$; e.g., AURKA, CCNA2, CCNE1, CDC2O, CCNB2, RRM2 and KIF2OA), cell cycle checkpoints $\left(\mathrm{P}=6.55 \times 10^{-7}\right.$; e.g., $C C N E 1, C D C 20$ and $C C N B 2)$, telomere maintenance $\left(\mathrm{P}=1.70 \times 10^{-6}\right.$; e.g., HIST1H2AC, HIST2H2AA3 and HISTIH2BD), DNA replication $\left(\mathrm{P}=3.52 \times 10^{-4}\right.$; e.g., RFC4 and POLE2) and metabolism of nucleotides ( $\mathrm{P}=4.04 \times 10^{-3}$; e.g., $T Y M S$ and RRM2) (Table III).

Analysis of transcription factors and genes associated with cancer. Transcription factor analysis of DEGs revealed that 21 transcription factors (e.g., ARNT, FOXP1 and HEYl) were 
Table I. Top 10 enriched GO terms in the Biological Process category for downregulated differentially expressed genes in bladder cancer.

\begin{tabular}{|c|c|c|c|}
\hline Term & $\mathrm{n}$ & P-value & Example genes \\
\hline GO: $0006936 \sim$ muscle contraction & 14 & $3.60 \times 10^{-5}$ & $C R Y A B, T A C R 2, M Y H 3, C A L D 1, V I P R 1 \ldots$ \\
\hline GO: $0003012 \sim$ muscle system process & 14 & $9.48 \times 10^{-5}$ & $C R Y A B, T A C R 2, M Y H 3, C A L D 1, V I P R 1 \ldots$ \\
\hline GO: $0030029 \sim$ actin filament-based process & 16 & $3.19 \times 10^{-4}$ & $D L C 1, M Y H 3, C A L D 1, N F 1, F L N A \ldots$ \\
\hline GO: $0001656 \sim$ metanephros development & 7 & $3.93 \times 10^{-4}$ & TCF21, BDNF, ITGA8, BCL2, HOXA11 .. \\
\hline GO: $0040012 \sim$ regulation of locomotion & 13 & $1.21 \times 10^{-3}$ & RTN4, DLC1, PRKCA, NF 1, SMAD3... \\
\hline GO: $0030334 \sim$ regulation of cell migration & 12 & $1.38 \times 10^{-3}$ & RTN4, DLC1, LAMA3, BCL2, NF1 .. \\
\hline GO: $0030336 \sim$ negative regulation of cell migration & 7 & $1.59 \times 10^{-3}$ & $D L C 1, B C L 2, N F 1, I L K, T G F B R 3 \ldots$ \\
\hline GO: $0030036 \sim$ actin cytoskeleton organization & 14 & $1.62 \times 10^{-3}$ & DLC1, CALD1, NF1, FLNA, CORO2B .. \\
\hline GO: $0060284 \sim$ regulation of cell development & 13 & $2.10 \times 10^{-3}$ & RTN4,NTF3, HOXA11,NF1,NLGN1.. \\
\hline GO: $0045449 \sim$ regulation of transcription & 79 & $2.24 \times 10^{-3}$ & ZNF383,LCOR,MAP3K13,RNF20, KCNH4.. \\
\hline
\end{tabular}

GO, gene ontology.

Table II. The two enriched Kyoto Encyclopedia of Genes and Genomes pathways for downregulated differentially expressed genes in bladder cancer.

\begin{tabular}{lcll}
\hline Term & $\mathrm{n}$ & P-value & \multicolumn{1}{c}{ Genes } \\
\hline hsa04510: Focal adhesion & 12 & $3.86 \times 10^{-3}$ & $\begin{array}{l}\text { PRKCA, LAMA3, ITGA8, BCL2, ILK, LAMC1, LAMB1, FLNC, FLNA, } \\
\text { PARVA, VCL, MYL9 } \\
\text { PRKCA, GNAI1, MYH3, CNKSR3, MYH11, MYH14, CLDN11, TJAP1, } \\
\text { hsa04530: Tight junction }\end{array}$ \\
\hline
\end{tabular}

hsa, homo sapiens.

Table III. Enriched Reactome pathways for downregulated and upregulated differentially expressed genes in bladder cancer.

\begin{tabular}{|c|c|c|c|}
\hline Term & $\mathrm{n}$ & P-value & Example genes \\
\hline \multicolumn{4}{|l|}{ Downregulated } \\
\hline REACT_17044: Muscle contraction & 5 & $2.46 \times 10^{-3}$ & TNNT3, DES, MYH3, DMD, TPM2 \\
\hline \multicolumn{4}{|l|}{ Upregulated } \\
\hline REACT_152: Cell cycle, mitotic & 49 & $3.93 \times 10^{-22}$ & CDC20, CCNB2, KIF23, E2F2, AURKA... \\
\hline REACT_1538: Cell cycle checkpoints & 17 & $6.55 \times 10^{-7}$ & CDC20, CCNB2, CHEK1, MCM2, UBE2C... \\
\hline REACT_7970: Telomere maintenance & 12 & $1.70 \times 10^{-6}$ & HIST1H2AC, HIST2H2AA3, HIST1H2BD, H2BFS, RFC4... \\
\hline REACT_383: DNA replication & 12 & $3.52 \times 10^{-4}$ & RFC4, POLE2, PSMC4, RFC2, PSMD10... \\
\hline REACT_1698: Metabolism of nucleotides & 9 & $4.04 \times 10^{-3}$ & $T Y M S, R R M 2, D T Y M K, D C K, C A D \ldots$ \\
\hline
\end{tabular}

significantly downregulated in bladder cancer tissues and 8 transcription factors (e.g., BCL3, EZH2 and FOXM1) were upregulated (Table VI).

Analysis of screening for genes associated with bladder cancer identified that 2 proto-oncogenes, DUSP26 and MEISI, were downregulated and 12 proto-oncogenes (e.g., AURKA, $C C N A 2$ and $C C N E 1$ ) were upregulated. Furthermore, 17 tumor suppressor genes (e.g., ARHGEF12, BLCAP and CHD5) were downregulated and 10 tumor suppressor genes (e.g., $B L M$, CHEK1 and CST6) were upregulated (Table VI).
PPI network and PPI sub-network analysis. A PPI network of DEGs was constructed (Fig. 1). The top five genes/proteins with the highest degree in the PPI network were $C C N A 2$, $B U B 1, C D C 20, C C N B 1$ and $M A D 2 L 1$, with degrees of 57, 53, 52,50 and 44, respectively.

The obtained core sub-network from the PPI network included 24 DEGs (Fig. 2). The CDC20 has the highest degree (degree, 18). A number of DEGs exhibited degrees $>10$, including CCNA2 (degree, 17), KIF11 (degree, 16), AURKA (degree, 15), NUSAP1 (degree, 15) and CCNB2 (degree, 14). 
Table IV. Top 10 enriched GO terms in Biological Process category for upregulated differentially expressed genes in bladder cancer.

\begin{tabular}{llll}
\hline Term & $\mathrm{n}$ & $\mathrm{P}$-value & \multicolumn{1}{c}{ Example genes } \\
\hline GO: $0007049 \sim$ cell cycle & 76 & $1.88 \times 10^{-29}$ & $C D C 20, C C N B 2, K I F 23, E 2 F 2, K I F C 1 \ldots$ \\
GO: $0000279 \sim$ M phase & 52 & $1.88 \times 10^{-29}$ & $C D C 20, C C N B 2, K I F 23, K I F C 1, P R C 1 \ldots$ \\
GO: $0022403 \sim$ cell cycle phase & 56 & $2.89 \times 10^{-28}$ & $C D C 20, C C N B 2, K I F 23, K I F C 1, P K M Y T 1 \ldots$ \\
GO: $0022402 \sim$ cell cycle process & 63 & $3.37 \times 10^{-27}$ & $C D C 20, C C N B 2, K I F 23, T T K, A U R K A \ldots$ \\
GO: $0000278 \sim$ mitotic cell cycle & 51 & $4.83 \times 10^{-26}$ & $C D C 20, C C N B 2, K I F 23, K I F C 1, T T K \ldots$ \\
GO: $0000280 \sim$ nuclear division & 40 & $7.79 \times 10^{-25}$ & $C D C 20, C C N B 2, K I F 23, K I F C 1, N E K 2 \ldots$ \\
GO: $0007067 \sim$ mitosis & 40 & $7.79 \times 10^{-25}$ & $C D C 20, C C N B 2, K I F 23, K I F C 1, P K M Y T 1 \ldots$ \\
GO: $0000087 \sim$ M phase of mitotic cell cycle & 40 & $1.56 \times 10^{-24}$ & $C D C 20, C C N B 2, K I F 23, P K M Y T 1, A U R K A \ldots$ \\
GO: $0048285 \sim$ organelle fission & 40 & $3.64 \times 10^{-24}$ & $C D C 20, C C N B 2, K I F 23, P T T G 1, C E P 55 \ldots$ \\
GO: $0051301 \sim$ cell division & 42 & $6.70 \times 10^{-22}$ & $C D C 20, C C N B 2, K I F 23, C K S 1 B, P R C 1 \ldots$ \\
\hline
\end{tabular}

GO, gene ontology.

Table V. The six enriched Kyoto Encyclopedia of Genes and Genomes pathways for upregulated differentially expressed genes in bladder cancer.

\begin{tabular}{|c|c|c|c|}
\hline Term & $\mathrm{n}$ & P-value & Example genes \\
\hline hsa04110: Cell cycle & 21 & $6.68 \times 10^{-11}$ & CDC20, CCNB2, E2F2, PKMYT1, TTK... \\
\hline hsa04114: Oocyte meiosis & 16 & $1.76 \times 10^{-7}$ & CDC20, CCNB2, SGOL1, PKMYT1, AURKA... \\
\hline hsa03030: DNA replication & 7 & $3.43 \times 10^{-4}$ & RFC4, POLE2, RFC2, MCM2, FEN1 .. \\
\hline hsa04115: P53 signaling pathway & 9 & $4.28 \times 10^{-4}$ & $C C N B 2, C C N E 2, C C N B 1, B I D, C C N E 1 \ldots$ \\
\hline $\begin{array}{l}\text { hsa04914: Progesterone-mediated } \\
\text { oocyte maturation }\end{array}$ & 9 & $2.05 \times 10^{-3}$ & $C C N B 2, C C N B 1, M A D 2 L 1, P L K 1, B U B 1 \ldots$ \\
\hline hsa05322: Systemic lupus erythematosus & 9 & $4.93 \times 10^{-3}$ & HIST1H2AC,HIST2H2AA3,CD86,HIST1H2BD,HIST1H2BK. \\
\hline
\end{tabular}

hsa, Homo sapiens.

Table VI. Transcription factors and genes associated with cancer in the differentially expressed genes in bladder cancer.

Terms

Genes

TF genes

Downregulated

ARNT, FOXP1, HEY1, HOXA11, HOXA3, HOXA9, ISL1, LMO3, MEIS1, NR1H3, NR1H4,

Upregulated NR3C2, POU3F 1, POU3F4, RORB, SMAD3, SP3, TCF21, YAF2, ZNF10, ZNF 174

Oncogenes

Downregulated

BCL3, EZH2, FOXM1, IRF1, RELB, TCEB3, TFDP1, XBP1

Upregulated

DUSP26, MEIS1

AURKA, BCL3, CCNA2, CCNE1, CEP55, DCUN1D1, FGFR1OP, HMMR, MAP3K8, MYB,

Tumor suppressors

Downregulated

ARHGEF12, BLCAP, CHD5, DLC1, FOXP1, MFHAS1, NDRG4, NF1, PACRG , PEG3, SCARA3,

Upregulated

BLM, CHEK1, CST6, ERRFI1, IRF1, MT1G, PLEKHG2, RASSF1, SLC9A3R1, TNFAIP3

$\mathrm{TF}$, transcription factor.

Furthermore, CDC20, CCNA2, CCNB2 and AURKA interacted with each other.
KEGG pathway enrichment analysis of the genes in the core sub-network revealed that DEGs were significantly involved in 
Table VII. Enriched Kyoto Encyclopedia of Genes and Genomes pathways for differentially expressed genes of protein-protein interaction sub-network.

\begin{tabular}{lcll} 
Term & $\mathrm{n}$ & \multicolumn{1}{c}{ P-value } & \multicolumn{1}{c}{ Genes } \\
\hline hsa04110: Cell cycle & 5 & $2.26 \times 10^{-5}$ & CCNB2, PLK1,CDC20,CCNA2, MCM6 \\
hsa04114: Oocyte meiosis & 4 & $5.10 \times 10^{-4}$ & CCNB2, PLK1, CDC20, AURKA \\
hsa04914: Progesterone-mediated oocyte maturation & 3 & $7.41 \times 10^{-3}$ & CCNB2, PLK1, CCNA2 \\
\hline
\end{tabular}

hsa, homo sapiens.

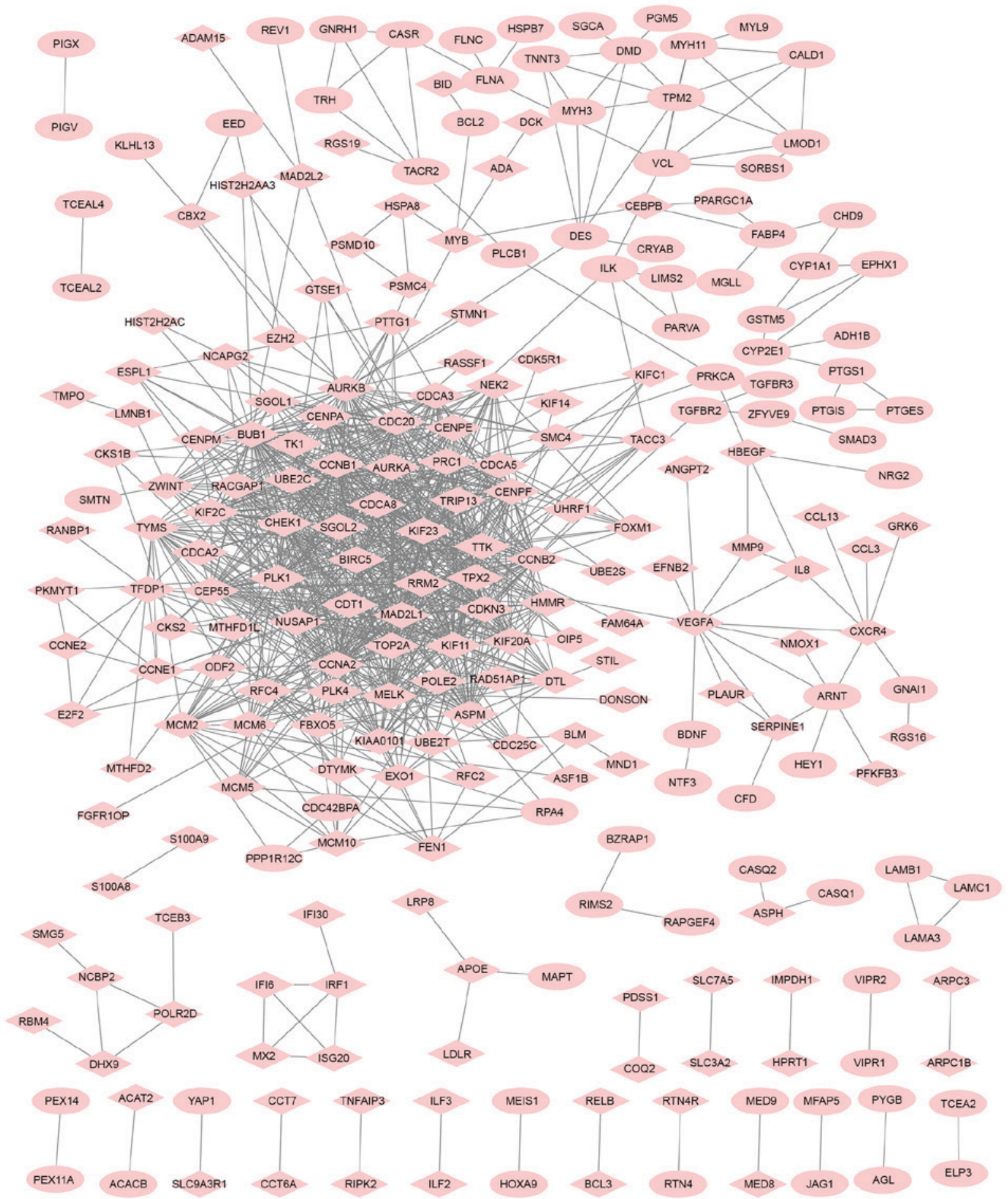

Figure 1. Protein-protein interaction network of differentially expressed genes. The diamond nodes represent upregulated differentially expressed genes and the round nodes represent downregulated differentially expressed genes. 


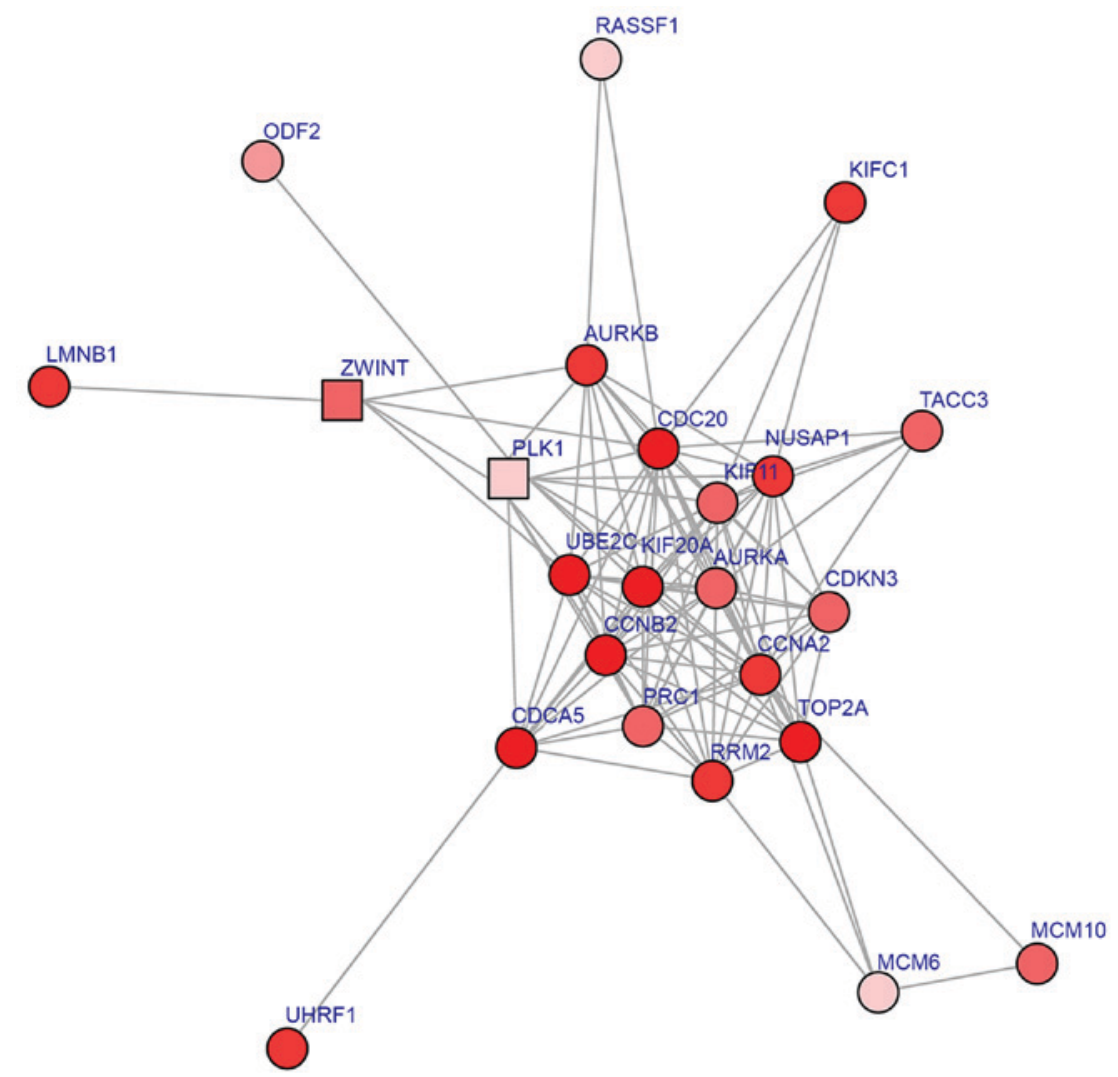

Figure 2. Protein-protein interaction sub-network of differentially expressed genes. The circles represent upregulated differentially expressed genes. The fold change of gene expression is presented through color (deeper color indicates higher fold change of gene expression). The square nodes represent the genes with lower importance in the sub-network.

cell cycle $\left(\mathrm{P}=2.26 \times 10^{-5} ; C C N B 2, P L K 1, C D C 20, C C N A 2\right.$ and $M C M 6)$, oocyte meiosis $\left(\mathrm{P}=5.10 \times 10^{-4} ; C C N B 2, P L K 1, C D C 20\right.$ and $A U R K A)$ and progesterone-mediated oocyte maturation $\left(\mathrm{P}=7.41 \times 10^{-3} ; C C N B 2, P L K 1\right.$ and $C C N A 2$; Table VII).

\section{Discussion}

In the present study, gene expression profiling was used to investigate the molecular mechanisms underlying bladder cancer. A set of 335 upregulated and 420 downregulated DEGs were identified between bladder cancer samples and normal controls. Analysis of the PPI sub-network demonstrated that 24 DEGs were obtained, and $C D C 20, C C N A 2, C C N B 2$ and AURKA had $>10$ degrees and interacted with each other. The pathway enrichment analysis revealed that these four genes were enriched in the cell cycle signaling pathway.

$C D C 20$ serves a key role in the spindle assembly checkpoint and is necessary for anaphase onset and cell cycle progression (21). The abnormal expression of spindle assembly checkpoint proteins during mitosis, including $C D C 20$, is associated with chromosome aneuploidy, and results in poor differentiation, tumor aneuploidy and poor prognosis (22). Kidokoro et al (23) demonstrated that p53 inhibits tumor cell growth by indirectly regulating the expression levels of $C D C 20$.

$C C N A 2$ and $C C N B 2$ encode cyclin and function as regulators of CDKs. In the present study, CCNA2 was identified as an upregulated proto-oncogene. Lu et al (24) and Lee et al (25) have demonstrated that $C C N A 2$ is upregulated in bladder cancer. Increased expression of $C C N A 2$ has been associated with poor prognosis for individuals with bladder cancer (26). Furthermore, in the present study, the KEGG pathway enrichment analysis identified that $C C N B 2$ was enriched in the p53 signaling pathway. It has been demonstrated that the expression level of CCNB2 is upregulated in bladder tumors during interphase and proteolysis (24), which is consistent with the results of the present study. Additionally, deletion of p53 in bladder epithelium has been demonstrated to lead to invasive cancer in a novel mouse model (27), indicating a key role for p53 in bladder cancer. Therefore, $C D C 20, C C N A 2$ and $C C N B 2$ may contribute to the development of bladder cancer.

AURKA, another upregulated proto-oncogene identified in the present study, encodes a cell cycle-regulated kinase (28). A previous study identified AURKA to be a biomarker for the detection of bladder cancer, due to AURKA aneuploidy resulting in chromosomal loss or gain (29). Genomic instability, which may be caused by checkpoint loss and perturbation of cell cycle control, results in the development of bladder cancer (30). A recent study demonstrated that AURKA is associated with the presence and grade of urothelial bladder cancer, suggesting a potential role as a diagnostic and prognostic biomarker (31). Hence, AURKA may serve a key role during the progression of bladder cancer.

However, the present study has a number of limitations. For example, the results of the present study were only predicted by bioinformatical analysis and must be further confirmed by experimental test. The studies should also be conducted using a larger sample sizes. These limitations are to be addressed in a further study. 
To conclude, the present study identified 420 downregulated and 335 upregulated DEGs. A number of important DEGs, including CDC20, CCNA2, CCNB2 and AURKA, may serve pivotal roles in the development of bladder cancer by regulating the cell cycle, as well as mutual interactions. These results provide a theoretical basis for a subsequent experimental study, and may contribute to an improved understanding of the molecular mechanisms that underlie bladder cancer.

\section{References}

1. Di Pierro GB, Gulia C, Cristini C, Fraietta G, Marini L, Grande P, Gentile V and Piergentili R: Bladder cancer: A simple model becomes complex. Curr Genomics 13: 395, 2012.

2. Ye F, Wang L, Castillo-Martin M, McBride R, Galsky MD, Zhu J, Boffetta P, Zhang DY and Cordon-Cardo C: Biomarkers for bladder cancer management: Present and future. Am J Clin Exp Urol 2: 1-14, 2014.

3. Sanchez-Carbayo M, Socci ND, Lozano J, Saint F and Cordon-Cardo C: Defining molecular profiles of poor outcome in patients with invasive bladder cancer using oligonucleotide microarrays. J Clin Oncol 24: 778-789, 2006.

4. Morrison CD, Liu P, Woloszynska-Read A, Zhang J, Luo W, Qin M, Bshara W, Conroy JM, Sabatini L, Vedell P, et al: Whole-genome sequencing identifies genomic heterogeneity at a nucleotide and chromosomal level in bladder cancer. Proc Nat Acad Sci USA 111: E672-E681, 2014.

5. Liu JY, Qian D, He LR, Li YH, Liao YJ, Mai SJ, Tian XP, Liu YH, Zhang JX, Kung HF, et al: PinX1 suppresses bladder urothelial carcinoma cell proliferation via the inhibition of telomerase activity and p16/cyclin D1 pathway. Mol Cancer 12: 148, 2013.

6. Florl AR and Schulz WA: Chromosomal instability in bladder cancer. Arch Toxicol 82: 173-182, 2008.

7. Zieger K, Wiuf C, Jensen KM, Ørntoft TF and Dyrskjøt L: Chromosomal imbalance in the progression of high-risk non-muscle invasive bladder cancer. BMC Cancer 9: 149, 2009.

8. Diboun I, Wernisch L, Orengo CA and Koltzenburg M: Microarray analysis after RNA amplification can detect pronounced differences in gene expression using limma. BMC Genomics 7: 252, 2006.

9. Dai M, Wang P, Boyd AD, Kostov G, Athey B, Jones EG, Bunney WE, Myers RM, Speed TP, Akil H, et al: Evolving gene/transcript definitions significantly alter the interpretation of GeneChip data. Nucleic Acids Res 33: e175, 2005.

10. Huang da W, Sherman BT and Lempicki RA: Systematic and integrative analysis of large gene lists using DAVID bioinformatics resources. Nat Protoc 4: 44-57, 2009.

11. Huang da W, Sherman BT and Lempicki RA: Bioinformatics enrichment tools: Paths toward the comprehensive functional analysis of large gene lists. Nucleic Acids Res 37: 1-13, 2009.

12. Ashburner M, Ball CA, Blake JA, Botstein D, Butler H, Cherry JM, Davis AP, Dolinski K, Dwight SS, Eppig JT, et al: Gene ontology: Tool for the unification of biology. The gene ontology consortium. Nat Genet 25: 25-29, 2000.

13. Kanehisa M and Goto S: KEGG: Kyoto encyclopedia of genes and genomes. Nucleic Acids Res 28: 27-30, 2000.

14. Croft D, Mundo AF, Haw R, Milacic M, Weiser J, Wu G, Caudy M, Garapati P, Gillespie M, Kamdar MR, et al: The Reactome pathway knowledgebase. Nucleic Acids Res 42 (Database Issue): D472-D477, 2014.

15. Matys V, Fricke E, Geffers R, Gößling E, Haubrock M, Hehl R, Hornischer K, Karas D, Kel AE, Kel-Margoulis OV, et al: TRANSFAC: Transcriptional regulation, from patterns to profiles. Nucleic Acids Res 31: 374-378, 2003.
16. Zhao M, Sun J and Zhao Z: TSGene: A web resource for tumor suppressor genes. Nucleic Acids Res 41 (Database Issue): D970-D976, 2013.

17. Chen JS, Hung WS, Chan HH, Tsai SJ and Sun HS: In silico identification of oncogenic potential of fyn-related kinase in hepatocellular carcinoma. Bioinformatics 29: 420-427, 2013.

18. Franceschini A, Szklarczyk D, Frankild S, Kuhn M, Simonovic M, Roth A, Lin J, Minguez P, Bork P, von Mering C and Jensen LJ: STRING v9.1: Protein-protein interaction networks, with increased coverage and integration. Nucleic Acids Res 41 (Database Issue): D808-D815, 2013.

19. Saito R, Smoot ME, Ono K, Ruscheinski J, Wang PL, Lotia S, Pico AR, Bader GD and Ideker T: A travel guide to Cytoscape plugins. Nat Methods 9: 1069-1076, 2012.

20. Beisser D, Klau GW, Dandekar T, Müller T and Dittrich MT: BioNet: An R-Package for the functional analysis of biological networks. Bioinformatics 26: 1129-1130, 2010.

21. Wang Y, Ji P, Liu J, Broaddus RR, Xue F and Zhang W: Centrosome-associated regulators of the $\mathrm{G} 2 / \mathrm{M}$ checkpoint as targets for cancer therapy. Mol Cancer 8: 8, 2009.

22. Choi JW, Kim Y, Lee JH and Kim YS: High expression of spindle assembly checkpoint proteins CDC20 and MAD2 is associated with poor prognosis in urothelial bladder cancer. Virchows Arch 463: 681-687, 2013.

23. Kidokoro T, Tanikawa C, Furukawa Y, Katagiri T, Nakamura Y and Matsuda K: CDC20, a potential cancer therapeutic target, is negatively regulated by p53. Oncogene 27: 1562-1571, 2008.

24. Lu Y, Liu P, Wen W, Grubbs CJ, Townsend RR, Malone JP, Lubet RA and You M: Cross-species comparison of orthologous gene expression in human bladder cancer and carcinogen-induced rodent models. Am J Transl Res 3: 8-27, 2010.

25. Lee SJ,Lee EJ, Kim SK, Jeong P, Cho YH, Yun SJ, Kim S, Kim GY, Choi YH, Cha EJ, et al: Identification of pro-inflammatory cytokines associated with muscle invasive bladder cancer; the roles of IL-5, IL-20, and IL-28A. PLoS One 7: e40267, 2012

26. Blaveri E, Simko JP, Korkola JE, Brewer JL, Baehner F, Mehta K, Devries S, Koppie T, Pejavar S, Carroll P and Waldman FM: Bladder cancer outcome and subtype classification by gene expression. Clin Cancer Res 11: 4044-4055, 2005.

27. Puzio-Kuter AM, Castillo-Martin M, Kinkade CW, Wang X, Shen TH, Matos T, Shen MM, Cordon-Cardo C and Abate-Shen C: Inactivation of $\mathrm{p} 53$ and Pten promotes invasive bladder cancer. Genes Dev 23: 675-680, 2009.

28. Medina-Aguilar R, Marchat LA, Arechaga Ocampo E, Gariglio P, García Mena J, Villegas Sepúlveda N, Martínez Castillo M and López-Camarillo C: Resveratrol inhibits cell cycle progression by targeting Aurora kinase A and Polo-like kinase 1 in breast cancer cells. Oncol Rep 35: 3696-3704, 2016.

29. Park HS, Park WS, Bondaruk J, Tanaka N, Katayama H, Lee S, Spiess PE, Steinberg JR, Wang Z, Katz RL, et al: Quantitation of Aurora kinase A gene copy number in urine sediments and bladder cancer detection. J Natl Cancer Inst 100: 1401-1411, 2008.

30. Wu X, Gu J, Grossman HB, Amos CI, Etzel C, Huang M, Zhang Q, Millikan RE, Lerner S, Dinney CP and Spitz MR: Bladder cancer predisposition: A multigenic approach to DNA-repair and cell-cycle-control genes. Am J Hum Genet 78: 464-479, 2006.

31. de Martino M, Shariat SF, Hofbauer SL, Lucca I, Taus C, Wiener HG, Haitel A, Susani M and Klatte T: Aurora a kinase as a diagnostic urinary marker for urothelial bladder cancer. World J Urol 33: 105-110, 2015.

This work is licensed under a Creative Commons Attribution-NonCommercial-NoDerivatives 4.0 International (CC BY-NC-ND 4.0) License. 